\title{
Multi-Carrier Burst Contention (MCBC): Scalable Medium Access Control for Wireless Networks
}

\author{
Bogdan Roman, Frank Stajano, Ian Wassell and David Cottingham
}

\begin{abstract}
With the rapid growth of WLAN capability for mobile devices such as laptops, handhelds, mobile phones and vehicles, we will witness WLANs with very large numbers of active nodes for which very efficient medium access control techniques will be needed to cope with high loads and mobility. We propose a high performance solution based on an innovative node elimination algorithm that uses short and unmodulated bursts of energy during contention - no data is exchanged. We also present a modified OFDM PHY layer, based on IEEE 802.11a, which allows sensing and bursting on individual subcarriers. We show that the protocol maintains a very low overhead and collision probability which lead to high and virtually constant network throughput at all analyzed network loads, even beyond 500 nodes. The protocol is validated by extensive simulation, comparing it against the IEEE 802.11a and SYN-MAC protocols.
\end{abstract}

\section{INTRODUCTION}

$\mathbf{I}$ $\mathrm{N}$ the future, we expect wireless communications to be in widespread use. We imagine a future where many people own wireless devices running information sharing or other network hungry applications in large highways or large conference halls or office buildings. Such networks may easily reach hundreds of active nodes. High mobility networks, such as vehicular networks, experience rapidly changing topologies imposing harsh conditions for wireless communication. Such factors make efficient access control to the medium a high priority. Distributed access mechanisms, particularly randomaccess through node contention, have proved to be a very efficient way to access the medium, especially in multi-hop or decentralized networks ${ }^{1}$. Ideally, the collision resolution mechanism should be low overhead and ensure a low collision probability, independent of network topology, and should scale with the network size to maintain a high throughput.

In this paper we present a high performance contentionbased MAC protocol, MCBC, which uses a novel node elimination algorithm to meet the above goals. Nodes send very short bursts, contender nodes being eliminated by referee nodes. Bearing some resemblance to binary countdown schemes and randomized leader election protocols, MCBC takes a different approach, exploiting the underlying OFDM PHY layer, distributing contention onto the frequency domain as well as the time domain within the same RF channel, also addressing the hidden node problem as early as the

The authors are with the Digital Technology Group, Computer Laboratory, University of Cambridge, United Kingdom (e-mail: abr28@cam.ac.uk).

The first author was supported by the Girton College at University of Cambridge, Ratiu Foundation UK and Felix Telecom Romania.

1802.11 infrastructure implementations also rely on node contention for medium access. Most manufacturers of 802.11 access point equipment do not implement the node-polling PCF mode due to various known problems [1]. contention session. In contrast to usual binary countdown or leader election schemes which need to exchange data frames, the bursts sent during contention carry no data and are very easy to produce. They are unmodulated OFDM subcarriers of short duration which we term blind energy bursts, thus providing very short delays, hence short contention windows and low overhead. Extensive simulation ${ }^{2}$ shows that the proposed algorithm offers a very low collision probability and scales with the network size, achieving a high throughput at all analyzed loads.

\section{RELATED WORK}

Landmark contention-based protocols employing RequestTo-Send/Clear-To-Send (RTS/CTS) and collision avoidance through binary backoff schemes are MACA [2] and MACAW [3], the latter forming the basis for the now widely adopted IEEE 802.11 [4]. Many variations on 802.11 have been developed that aim to improve its throughput. Examples are adaptive contention window initialization or resizing [5], [6], non-uniform or adaptive counter increase/decrease [7], [8] and estimating network topology parameters [9], [10]. As first shown by Bianchi [11], the only way to make the 802.11 DCF scalable with the network size is by using adaptive methods, which introduces the adaptation problem for networks with variable topologies ${ }^{3}$. Such solutions can introduce frame delays and unfairness in networks with high mobility and do not scale well for very large networks. A scheme employing unmodulated bursts is introduced by Sobrinho and Krishnakumar [12], aiming to obtain QoS at the MAC level with the burst duration being directly proportional to the node's elapsed waiting time. This however would introduce unacceptable overhead in medium or large networks. A different scheme which uses selective OFDM subcarriers, called OFDM spectrum pooling, is proposed by Weiss and Friedrich [13], the purpose being to sense licensed spectrum usage in cognitive radio networks.

Hybrid TDMA solutions employing contention in each time slot appeared in the ABROAD [14] and AGENT [15] protocols and more recently in protocols for sensor networks [16]-[18]. Most such solutions assign and maintain TDMA frame parameters, which is suitable for sensor networks where the goal is

\footnotetext{
${ }^{2}$ The analytical model including collision probability, network throughput and delay analysis and protocol correctness theorems are excluded due to space limitations. Also excluded are the results of different contention parameter values. All such material is available on request.

${ }^{3}$ In adaptive MAC schemes there is a clear trade off between adaptation time and tracking ability which does not play well with variable topologies. The complexity is also usually high as is the power consumption.
} 
mainly to minimize power consumption and where topology changes occur rarely. However, they scale poorly with the network size or variable topologies because of the overhead and adaptation time needed to recompute frame parameters. A solution is to relax the TDMA constraints to allow variable or unspecified frame lengths and use slot reservation through contention. Such a hybrid protocol that also employs feedback from the network in the contention phase is FPRP [19], which is shown to yield low collision probability. However, it requires a significant amount of overhead because of numerous control frame exchanges per contention round, becoming unstable in larger networks. ADHOC MAC [20] uses a reliable reservation ALOHA (RR-ALOHA) protocol to establish slotted medium access with high flexibility. However, the nodes need to constantly send frame information thus increasing the overhead and lowering the throughput. The CSMA/CP [21] protocol is based on carrier sensing and binary countdown ${ }^{4}$ on a separate control channel. This is however inefficient since an entire channel is dedicated to contention. A common problem of binary countdown schemes applied in wireless networks is that of poor performance in multi-hop scenarios, hidden nodes increasing the collision probability considerably, so feedback schemes similar to RTS/CTS become necessary. A more recent solution based on binary countdown which does not use separate channels or RTS/CTS is SYN-MAC [22] which is included for comparison in this paper. The random binary countdown slots of SYN-MAC are followed by a feedback slot to address the hidden node problem.

\section{THE MCBC PROTOCOL}

Our MCBC protocol follows the IEEE 802.11 model [4] as in Fig 1. The major difference is that synchronized contention windows of fixed duration precede every RTS transmission. A node contends only at predefined times and only if the channel is sensed idle during a distributed inter-frame space (DIFS) interval. Synchronization can be achieved by listening to the medium for contention bursts, data and control frames or beacons or externally, e.g. with the help of a GPS $\mathrm{clock}^{5}$ which is readily available in vehicular networks. The CWs are equally spaced in time with period $T_{c}$ which includes a $\mathrm{CW}$, an RTS frame and a DIFS interval. The contention winner sends an RTS frame and waits for a CTS reply from the destination. If the CTS is not received then nodes may start contending at the next $T_{c}$, thus ensuring a rapid recovery from collisions. In MCBC, channel reservation can be represented as multiples of $T_{c}$, reducing the duration field size of the MAC header.

An important function of RTS/CTS, besides quick collision recovery and multi-hop channel reservation is that it addresses the hidden node problem, since the CTS reply is heard two hops away by all the receiver node's neighbors thus rendering

\footnotetext{
${ }^{4}$ In binary countdown schemes, time is slotted into $k$ slots and contending nodes generate a $k$-bit number, listening and transmitting in slots corresponding to $0 \mathrm{~s}$ and $1 \mathrm{~s}$ respectively of the $k$-bit number. A node gives up when a higher number is detected.

${ }^{5}$ In case of GPS clock sync, we note the existence of low cost off-the-shelf products with $15 \mathrm{~ns}$ accuracy [23]. Clock drift due to loss of GPS signal has been shown to be less than 90 ns per hour [24] which can be neglected since MCBC uses a sync guard time of $\geq 1 \mu \mathrm{s}$.
}

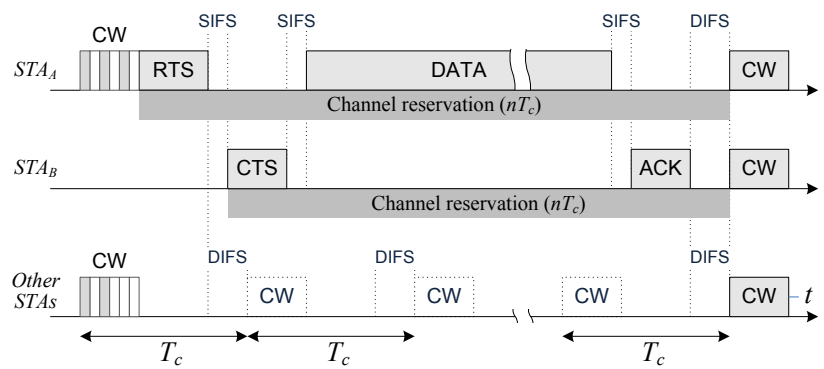

Fig. 1. MCBC: $\mathrm{STA}_{\mathrm{A}}$ wins the contention and sends data to $\mathrm{STA}_{\mathrm{B}}$

them silent for the duration of the data transmission. One important advantage of MCBC is that it mitigates the hidden node problem in the contention session, as we later explain. Using fixed size data frames enables MCBC to function without RTS/CTS thus gaining considerably in network throughput, especially at high bit rates. We call this mode MCBC-nRC (MCBC no RTS/CTS), which is similar to hybrid TDMA schemes employing contention in every time slot, such as SYN-MAC [22].

\section{A. OFDM PHY with Contention Subcarriers}

The MCBC PHY is based on the IEEE 802.11a [25] OFDM PHY. MCBC enhances the OFDM PHY so that individual subcarriers can be activated and sensed during contention. This bears some resemblance to OFDM spectrum pooling [13] used in cognitive radio. From here on, we define a contention subcarrier as an unmodulated signal of short duration carrying no data (a blind energy burst) on the frequency of one of the OFDM subcarriers that are within the same channel. The enhancement consists in the addition of two blocks: the contention subcarrier activation (CSA) in the transmitter and contention subcarrier sensing (CSS) in the receiver.

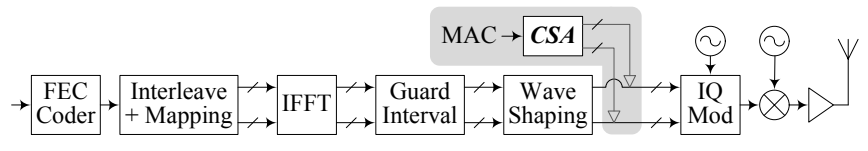

Fig. 2. OFDM PHY transmitter. 3-state buffers omitted for simplicity.

Fig. 2 shows the enhanced OFDM PHY transmitter. To transmit a blind energy burst on one subcarrier, MCBC exploits the properties of the Fast Fourier Transform (FFT). A constellation with non-zero values at subcarrier $j$ and zero at all subcarriers $i \neq j$ given as input to the Inverse FFT (IFFT) block will yield a signal with the energy concentrated on subcarrier $j$. During contention, MCBC uses only a subset of $n_{f} \leq 8$ contention subcarriers out of the total of 52 subcarriers used during data transmission, and activates only one contention subcarrier in a contention or feedback slot, as we will see in the next sub-section. For contention subcarrier activation, the outputs of the IFFT block corresponding to each of the $n_{f}$ subcarriers are hard-wired into the CSA block and all previous PHY blocks are bypassed thus achieving a very low delay. The reverse process happens similarly in the receiver, where the output of the FFT block is used directly by the CSS block to detect active subcarriers and a simple 
bitmask of $n_{f}$ bits is then passed to the MAC. The bitmask is 1 at all subcarrier indexes where an active subcarrier was detected and 0 at the others. In this way, the delay in activating or sensing contention subcarriers is reduced, giving a low contention overhead ${ }^{6}$.

MCBC transmits no OFDM preamble during contention bursts and thus frequency drift could be a problem. The IEEE 802.11a standard specifies a transmitter center frequency tolerance of $\max \pm 20 \mathrm{ppm}(104 \mathrm{KHz}$ tolerance at $5.2 \mathrm{GHz})$. Doppler shift is negligible (e.g. at $100 \mathrm{mph}(161 \mathrm{Km} / \mathrm{h})$ and $5.2 \mathrm{GHz}$, the doppler shift is only $0.76 \mathrm{KHz}$ ). MCBC alleviates frequency drift first by spacing the $n_{f} \leq 8$ contention subcarriers so that any non-zero contribution from adjacent contention subcarriers (a.k.a. FFT leakage) is minimized. Fig. 3 shows the spectrum of 4 contention subcarriers spaced with 10 times the normal subcarrier spacing. The sinc shape is the result of time windowing with a rectangular window. One can see that the side lobes rate of decay is very low and multiple simultaneous bursts may yield false positives. The larger spacing between the $n_{f}$ contention subcarriers allows us to address this problem by using a different time window that has a much higher side lobe attenuation and a wider main lobe, such as the Kaiser window [26] with $\alpha>8$. This signal is programmed into the CSA block so the delay and implementation complexity remain unchanged.

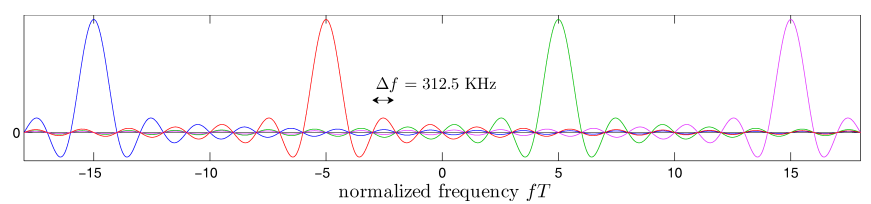

Fig. 3. Received spectrum of $n_{f}=4$ perfectly synchronized orthogonal contention subcarriers spaced by 10 times the normal subcarrier spacing used during data transmission with 52 subcarriers which is $312.5 \mathrm{KHz}$.

In fast fading or frequency selective fading environments where some subcarriers might be rendered unavailable, a contention subcarrier can be represented by a group of subcarriers spread over the OFDM spectrum. This scheme adds no complexity to the MAC or PHY implementation since the waveforms generated by the CSA block can activate any number of subcarriers. We note that the functionality of the algorithm presented here is unaffected by such a scheme.

\section{B. MAC Collision Resolution}

Each contention window has a fixed length of $n_{s}$ slot pairs, which we will call rounds, each comprising a contention slot followed by a feedback slot. During contention, a node can be in one of the following states: contender, nominee

\footnotetext{
${ }^{6}$ The short inter-frame space defined in 802.11a which is used between data transmissions SIFS $=a R x R F$ Delay $+a R x P L C P D e l a y+$ $a M A C$ ProcessingDelay $+a R x T x$ TurnaroundTime $=16 \mu \mathrm{s}$ does not apply in MCBC during contention. A better comparison is aSlotTime $=$ aCCATime $+a R x$ TxTurnaroundTime + aAirPropagationTime + $a M A C$ ProcessingDelay $=4+2+1+2=9 \mu s$. In MCBC, clear channel assessment (CCA), in our case subcarrier sensing, can be performed more quickly, and the RxTx turnaround and MAC processing delays are shorter due to the bypassing of several PHY blocks and the much simpler processing performed by the MAC during contention (no frame data processing or response data preparation). MCBC uses a slot time of 7 or $8 \mu \mathrm{s}$.
}

or referee. Nodes with data to send start as contenders, while the others are referees. In each round, a fraction of contenders are randomly promoted to nominees, meaning they can compete. The nominees who win the round are promoted to contenders for the next round, while those who lose become referees. The nominees who win the last round initiate their data transmission. Thus, the primary goal of the algorithm is to maximize the probability that there is only one winning nominee in the last round. The algorithm state diagram is given in Fig. 4, followed by its description and a complete example.

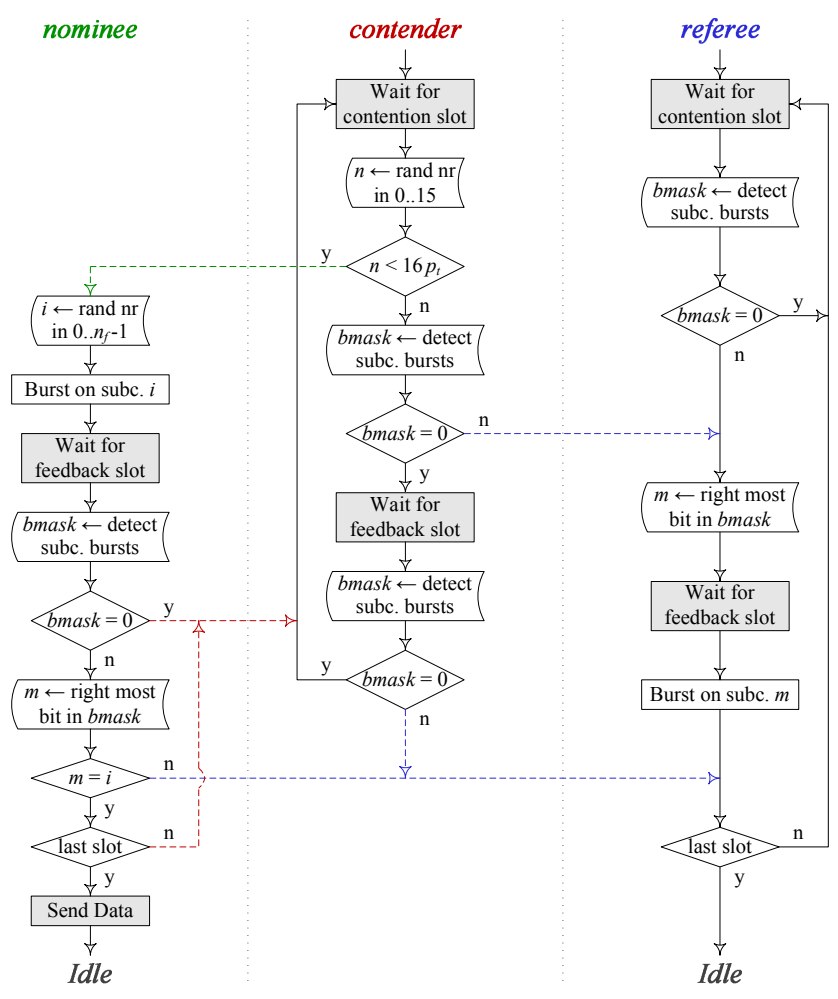

Fig. 4. Contention algorithm running in each node. The node starts from Idle as contender if it has data to send, otherwise as referee. Dashed lines represent transitions between contender, nominee and referee states. For clarity, several diagram elements were duplicated.

In the contention slot, contenders become nominees with probability $p_{t}$. Nominees then transmit a burst on a random ${ }^{7}$ contention subcarrier $i$ out of the $n_{f}$ predefined ones. At the same time, the other nodes listen for bursts on the $n_{f}$ contention subcarriers and, if any are detected, they become referees and record the highest numbered contention subcarrier a burst was heard on as $m$ (i.e. the right most non-zero bit index in the subcarrier bitmask of $n_{f}$ bits). In the feedback slot, referees activate subcarrier $m$. At the same time, the nominee nodes which detect their chosen subcarrier (i.e. the right most non-zero bit index of the received bitmask is $i$ ) change status to contender, whilst the others will change status to referee. The contention proceeds into the next round until the last feedback slot $n_{s}$ after which all standing nominees initiate their data transmission.

Fig. 5 shows a contention session example for a one-hop

\footnotetext{
${ }^{7}$ Hardware wise, a longer sequence of random bits is generated before contention and then groups of 4 and 3 bits are extracted.
} 


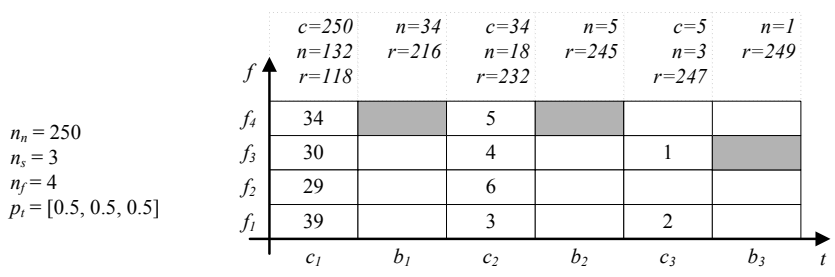

Fig. 5. Contention session example; $c, r$ and $n$ are the number of contenders, referees and nominees respectively. The shaded cells denote the contention subcarrier activated by referees in the feedback slots.

network of 250 nodes at saturation (i.e. all nodes have data to send). The $n_{n}=250$ contenders become nominees with probability $p_{t}=0.5$. Each of the resulting 132 nominees activates a random contention subcarrier $f_{i}$. At this point, the remaining 118 contenders become referees by detecting a contention subcarrier. In feedback slot $b_{1}$, the referees activate the subcarrier from the right most detected subcarrier index in $c_{1}$, in this case $f_{4}$. The 34 nominees that activated $f_{4}$ in $c_{1}$ are thus selected as round winners and are promoted to contenders while the remaining 98 become referees. The contention continues similarly in $c_{2}$ with 34 contenders and 216 referees. In $c_{3}, 3$ out of 5 contenders become nominees and activate a subcarrier. In $b_{4}$ referees reply on the right most detected subcarrier index, $f_{3}$, and we are left with one winning nominee that will initiate its data transmission.

We note that if there is no reply in the feedback slot because there were no nominees in the contention slot (no referees in the contention slot) then everybody wins, i.e. all contenders keep their status (all nominees are promoted to contenders). This ensures the protocol's correctness that there will always be at least one winning nominee at the end of the contention, and a unique round winner will be the overall contention winner as well.

As we can see, the number of contenders decreases exponentially, at the end of the contention session expecting an average of approximately $\left\lceil n_{n} p_{t}^{n_{s}} n_{f}-n_{s}\right\rceil$ contenders for a fixed $p_{t}$.

Fairness in $\mathrm{MCBC}$ is ensured since each station uses the same contention parameters for every contention session. There will be no stations having priority over the rest.

\section{Multi-hop Network}

The replies from the referees in the feedback slots are heard by two-hop neighbors as well. This effectively enables MCBC to address the hidden node problem in the contention session, allowing it to function without RTS/CTS, thus gaining considerable throughput. A downside however is reduced spatial reuse. An example is given in Fig. 6, where a node cannot hear a neighbor two hops away. It can be seen that the hidden node problem is eliminated although in scenario $b$ ) spatial reuse is reduced because either node 2 or node 4 may transmit, but not both.

\section{Simulation Results}

MCBC uses the same parameters as 802.11a with a few exceptions. The common parameters for the protocols are

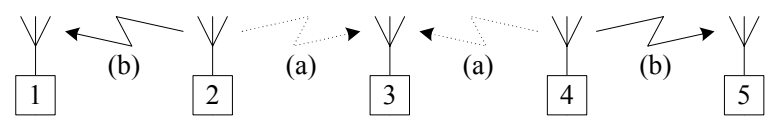

Fig. 6. Two separate network scenarios: a) node 2 and node 4 have data for node 3 ; b) node 2 has data for node 1 and node 4 has data for node 5 . In both scenarios, nodes 1,3 and 5 are referees.

outlined in Table $\mathrm{I}^{8}$. The PHY changes outlined in section III-A allow for very short delays in activating and detecting subcarriers, hence a lower slot duration of $t_{s}=7 \mu \mathrm{s}$ which includes RxTx turnaround, MAC and PHY processing delay, sync guard and propagation delay. The DIFS has been chosen as SIFS $+t_{s}$. Unless otherwise specified, the following parameters were used: $n_{s}=3$ contention rounds, $n_{f}=6$ contention subcarriers out of 52, giving a large subcarrier spacing of $10 \Delta f$ to minimize FFT leakage (see section III-A) and $p_{t}=[2 / 16,13 / 16,13 / 16]$ as the probability of transmission vector for the 3 contention slots. For SYN-MAC, DIFS is equal to SIFS, there is no RTS/CTS exchange and the number of contention slots was fixed at $k=10$, the value found to be optimal by the SYN-MAC authors.

TABLE I

SIMULATION PARAMETERS.

\begin{tabular}{r||c|c|c|c}
\hline & 802.11 & SYN-MAC & MCBC & MCBC-nRC \\
\hline DATA frame (bits) & 8184 & 8184 & 8184 & 8184 \\
PLCP preamble ( $\mu s)$ & 16 & 16 & 16 & 16 \\
PLCP header9 (bits) & 46 & 46 & 46 & 46 \\
MAC header (bits) & 272 & 272 & 272 & 272 \\
RTS (bits) & 160 & $\mathrm{n} / \mathrm{a}$ & 160 & $\mathrm{n} / \mathrm{a}$ \\
CTS (bits) & 112 & $\mathrm{n} / \mathrm{a}$ & 112 & $\mathrm{n} / \mathrm{a}$ \\
ACK (bits) & 112 & 96 & 112 & 96 \\
SIFS ( $\mu s)$ & 16 & 16 & 16 & 16 \\
DIFS $(\mu s)$ & 34 & 16 & 23 & 16 \\
Prop. delay $\delta(\mu s)$ & 1 & 1 & 1 & 1 \\
\hline
\end{tabular}

We used a custom built simulator written in GNU C 99, weighing $\sim 3000$ lines of code and optimized for speed, that closely follows all protocol details for each node, including turnaround times, propagation delays, guard times etc. All results were obtained with a $95 \%$ confidence interval lower than $10^{-3}$. The results of the simulator were verified up to 50 nodes using the NS-2 simulator and matched the ones obtained in [11], [27] and [22]. We also conducted real-world experiments for 1 to 5 nodes with Atheros AR5002X [28] based 802.11a cards and the simulation results were found to be more than $99.7 \%$ accurate.

We considered a one-hop network at saturation (asymptotic conditions) in an ideal channel. The throughput and delay were measured at the MAC level. As first noted in [11], the only way to make IEEE 802.11 DCF scalable with the network

${ }^{8}$ The $802.11 \mathrm{~b}$ standard for HR/DSSS specifies a PLCP preamble (header) of 72 bits (48 bits) transmitted at 1 Mbps DBPSK (2 Mbps DQPSK) fixed rate and a minimum wait time of $S I F S=10 \mu \mathrm{s}$ for any data exchange. Although SYN-MAC exchanges data during contention, it assumes a lower wait time of $5 \mu \mathrm{s}$ and only a PLCP header of 48 bits transmitted at $11 \mathrm{Mbps}$ and no sync guard time or propagation delay. Real OFDM PHY and MAC parameters would greatly decrease the performance of SYN-MAC. So, during contention, we have used the values specified by the SYN-MAC authors.

${ }^{9}$ The first 24 bits of the PLCP header are always transmitted at $6 \mathrm{Mbps}$. Also, a variable number of pad bits are added to the 46 bits to bring the PHY frame duration to a multiple integer of one OFDM symbol $(4 \mu s)$. 
size is to employ adaptive methods. Increasing the minimum contention window size $W$ of the 802.11 DCF increases its network throughput at higher loads but decreases it at low loads. These findings were also confirmed by our simulations. The standard specifies $W=16$. MCBC and SYN-MAC are non-adaptive protocols so fixed contention parameters were used for all three protocols.

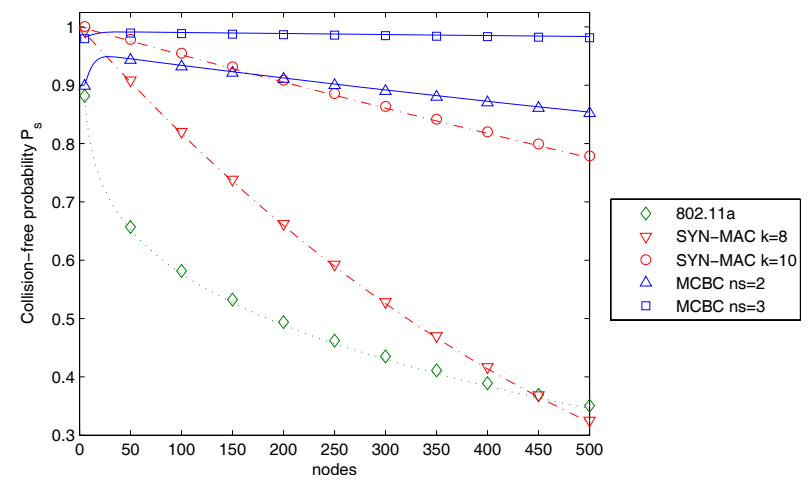

Fig. 7. Collision-free probability. Analysis (lines) and simulation (symbols).

Fig. 7 shows the collision-free probability $P_{s}$. Because of the fast exponential decrease in number of contender nodes, the MCBC scheme with $n_{s}=3$ rounds and $n_{f}=6$ contention subcarriers provides a very high $P_{s}$, being above 0.98 (i.e. less than $2 \%$ collisions) for the whole range of nodes. SYN-MAC also provides a high collision-free probability for any $k \geq 10$, especially at very low loads. The inefficiency of the binary backoff algorithm of 802.11 clearly stands out, $P_{s}$ dropping below 0.7 (30\% collisions) very quickly.

A high $P_{s}$ is many times irrelevant to throughput and delay performance, the contention overhead and collision recovery sometimes being more important. This is the case for SYNMAC and 802.11. The efficiency of RTS/CTS which quickly recovers after collisions, effectively makes the throughput of 802.11 less sensitive to $P_{s}$ as seen in Fig. 8. In the case of SYN-MAC, even though the collision-free probability is high and almost constant, the contention overhead added by the $k=10$ contention slots is anything but negligible, needing numerous RxTx turnarounds and PHY headers, causing the throughput to drop considerably. When no RTS/CTS (or similar) scheme is used, the duration of a collision becomes important and comparable to the duration of a successful transmission, making the throughput almost directly proportional to $P_{s}$. This can be noticed for 802.11a basic (i.e. 802.11a without RTS/CTS), SYN-MAC and MCBC-nRC especially in Fig. 9 and Fig. 10.

Thanks to the nature of the contention which does not send any data and the short duration of the bursts, MCBC (which uses RTS/CTS) achieves a virtually constant throughput. Aided by a high $P_{s}$ and low contention overhead, it then becomes advantageous to drop the RTS/CTS scheme and MCBC-nRC reaches considerably higher throughputs, up to $23 \%$ higher at low loads (Fig. 8) and up to $42 \%$ higher at high loads (Fig. 9) compared to 802.11a at 24 Mbps. We note that without RTS/CTS, MCBC-nRC can still effectively mitigate the hidden node problem. An obvious disadvantage, however,

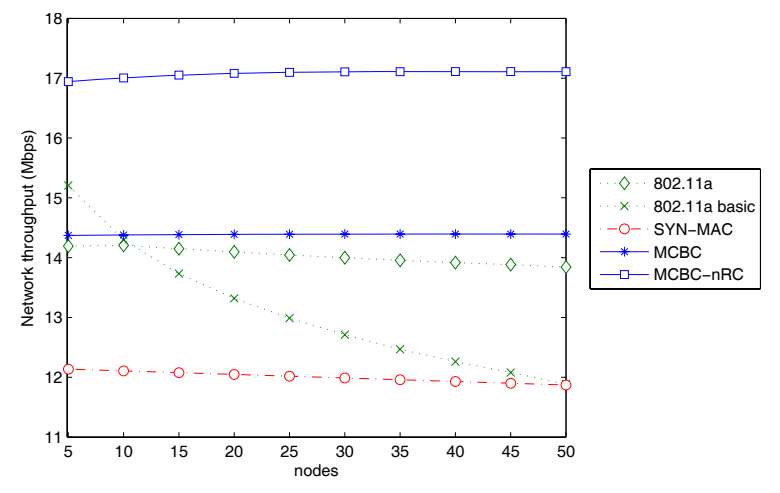

Fig. 8. Network throughput at low loads for a channel bitrate of 24 Mbps.

is that data frames of fixed size should be employed, since two-hop channel reservation is no longer possible. A common solution, also deployed in IEEE 802.11e, is to aggregate packets to maintain a bigger MAC frame size for increased efficiency.

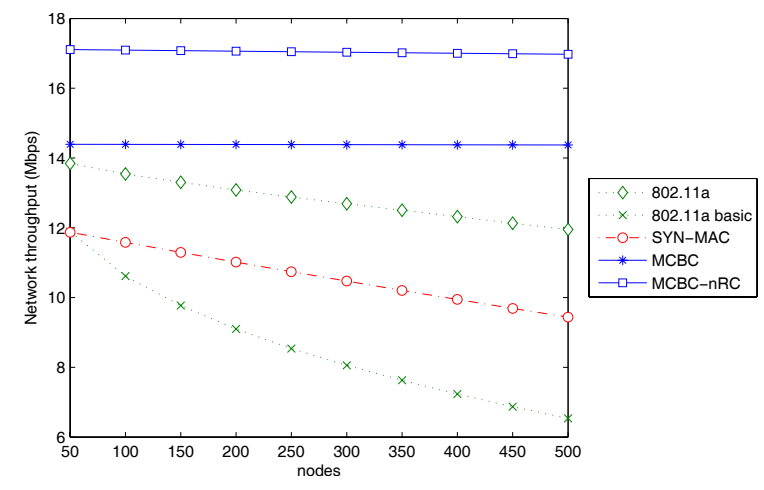

Fig. 9. Network throughput at high loads for a channel bitrate of $24 \mathrm{Mbps}$.

At high loads, shown in Fig. 9 and Fig. 10, the dependence on $P_{s}$ becomes more evident. An interesting, but expected result is that the throughput gain of MCBC-nRC increases with the channel bit rate. As the bit rate increases, the duration of data frames decreases while inter-frame spaces remain constant. Thus, the two SIFS intervals that were part of RTS/CTS help gain even more throughput, MCBC-nRC reaching a considerable 68\% gain compared to $802.11 \mathrm{a}$ at $54 \mathrm{Mbps}$, also reflected in the delay results in Fig. 11. This would not have been possible without a high $P_{s}$ and short contention windows.

Analysis and simulations showed that $\mathrm{MCBC}$ is able to scale even with networks with thousands of nodes. At 1000 nodes the collision-free probability is $P_{s}=0.9757$ and at 2000 nodes $P_{s}=0.9638$ giving a network throughput of 22.484 Mbps (29.017 Mbps) and 22.414 Mbps (28.663 Mbps) and an average per-node frame delay of $364 \mathrm{~ms}(282 \mathrm{~ms})$ and $730 \mathrm{~ms}$ (571 ms) respectively for MCBC (MCBC-nRC). However, besides impracticality, the average per-node throughput at such extreme loads may drop below acceptable limits.

\section{CONCLUSion AND FUture WorK}

MCBC is a random access MAC protocol which uses a novel contention mechanism employing a rapidly converging 


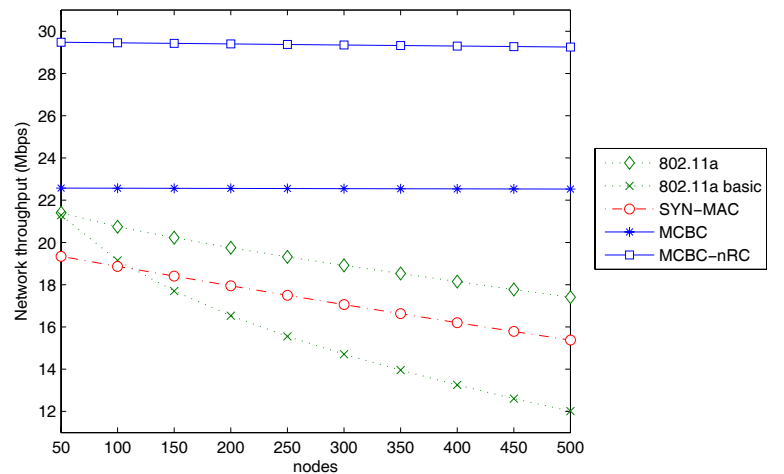

Fig. 10. Network throughput at high loads for a channel bitrate of $54 \mathrm{Mbps}$.

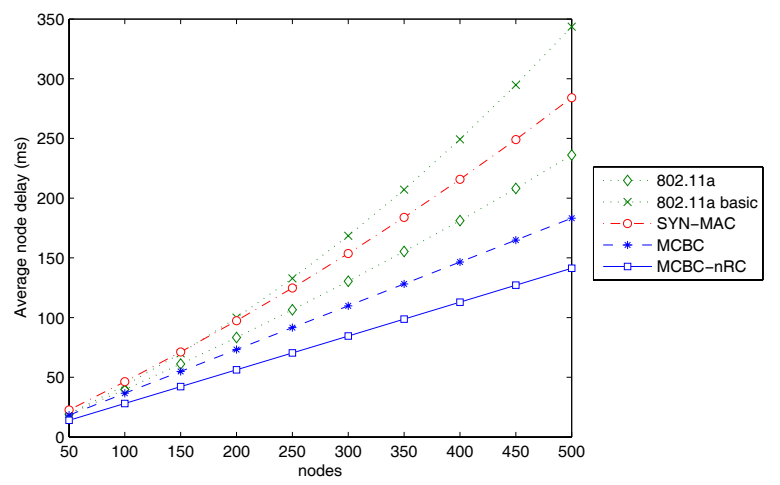

Fig. 11. Average node delay at high loads for a channel bitrate of $54 \mathrm{Mbps}$.

collision resolution algorithm in which nodes compete and are eliminated by their one-hop neighbors using short, unmodulated energy bursts. We have presented a modified OFDM PHY layer that allows sensing and bursting on individual subcarriers with little overhead. The protocol provides considerable gains in network throughput compared to existing and proposed solutions and scales almost arbitrarily with the network size. Since the network topology remains constant for the duration of a contention session ( $42 \mu s$ for $n_{s}=3$ ) and since the collision resolution algorithm is non-adaptive (i.e. does not depend on topology or outcome of any previous transmission attempts), we consider MCBC to be topology transparent. This allows it to be used under challenging conditions where there is a highly variable number of nodes and high mobility. The short duration of the contention sessions and the lack of data exchange also make the MCBC contention algorithm suitable for collaborative networks (a.k.a. node diversity schemes) as well as real-time leader election protocols.

We are currently implementing an MCBC prototype in hardware. Reliable Quality of Service, a feature we had in mind when designing $\mathrm{MCBC}$, is currently work in progress and the subject of a future paper. We are also investigating adaptive versions of the algorithm that will allow reducing the number of contention rounds or contention subcarriers.

\section{ACKNOWLEDGMENTS}

We thank our colleagues in the Digital Technology Group for a stimulating environment in which to conduct this research, particularly Mbou Eyole, Ioannis Chatzigeorgiou and Andy Rice for their advice, reviews and insightful discussions.

\section{REFERENCES}

[1] S. Mangold, S. Choi, G. Hiertz, and O. Klein, "Analysis of IEEE 802.11e for QoS Support in Wireless LANs," IEEE Wireless Commun. Mag., vol. $10(6), 2003$.

[2] P. Karn, "MACA A New Channel Access Protocol for Packet Radio," in ARRL/CRRL Amateur Radio 9th Comput. Net. Conf., 1990.

[3] V. Bharghavan, A. Demers, S. Shenker, and L. Zhang, "MACAW: A Media Access Protocol for Wireless LAN's," Proc. ACM SIGCOMM'94, 1994.

[4] 802.11-1999 (R2003), Wireless LAN Medium Access Control and Physical Layer Specifications, IEEE Std., 1999, Reaffirmed June 2003.

[5] C. Wang, B. Li, and L. Li, "A New Collision Resolution Mechanism to Enhance the Performance of IEEE 802.11 DCF," IEEE Trans. Veh. Technol., vol. 53, no. 4, 2004.

[6] N. O. Song, B. J. Kwak, J. Song, and L. E. Miller, "Enhancement of IEEE 802.11 Distributed Coordination Function with Exponential Increase Exponential Decrease Backoff Algorithm,' in IEEE VTC'03 Spring, 2003.

[7] N. Choi, Y. Seok, Y. Choi, S. Kim, and H. Jung, "P-DCF: enhanced backoff scheme for the IEEE 802.11 DCF," in IEEE VTC'05 Spring, 2005.

[8] Y. Li, L. Ke-Ping, Z. Wei-Liang, and C. Qian-Bin, "A Novel Random Backoff Algorithm to Enhance the Performance of IEEE 802.11 DCF," Springer J. Wireless Personal Commun., vol. 36, 2006.

[9] F. Cali, M. Conti, and E. Gregori, "IEEE 802.11 Protocol: Design and Performance Evaluation of an Adaptive Backoff Mechanism," IEEE J. Sel. Areas Commun., vol. 18, no. 9, 2000.

[10] Y. Kwon, Y. Fang, and H. Latchman, "A novel MAC protocol with fast collision resolution for wireless LANs," in IEEE INFOCOM03, 2003.

[11] G. Bianchi, "Performance Analysis of the IEEE 802.11 Distributed Coordination Function," IEEE J. Sel. Areas Commun., vol. 18 (3), 2000.

[12] J. Sobrinho and A. Krishnakumar, "Quality-of-Service in Ad Hoc Carrier Sense Multiple Access Wireless Networks," IEEE J. Sel. Areas Commun., vol. 17, no. 8, 1999.

[13] T. Weiss and F. Jondral, "Spectrum pooling: An Innovative Strategy for the Enhancement of Spectrum Efficiency," IEEE Commun. Mag., vol. 42, pp. 8-14, Mar. 2004

[14] I. Chlamtac, A. Myers, V. R. Syrotiuk, and G. Zaruba, "An Adaptive Medium Access Control (MAC) Protocol For Reliable Broadcast In Wireless Networks," in IEEE Intl. Conf. on Commun., vol. 3, 2000.

[15] A. Myers, G. Zaruba, and V. R. Syrotiuk, "An Adaptive Generalized Transmission Protocol for Ad Hoc Networks," ACM Mob. Netw. and Apps., vol. 7, no. 6, pp. 493-502, Dec. 2002.

[16] V. Rajendran, K. Obraczka, and J. Garcia, "Energy-efficient, collisionfree medium access control for wireless sensor networks," in ACM Conf. on Emb. Netw. Sensor Sys., 2003.

[17] I. Rhee, A. Warrier, M. Aia, and J. Min, "Z-MAC: a Hybrid MAC for Wireless Sensor Networks," in ACM Conf. on Emb. Netw. Sensor Sys., 2005.

[18] S. B. Eisenman and A. T. Campbell, "Structuring Contention based Channel Access in Wireless Sensor Networks," in IPSN'06, 2006.

[19] C. Zhu and M. S. Corson, "A Five-Phase Reservation Protocol (FPRP) for Mobile Ad Hoc Networks," in IEEE INFOCOM'98, vol. 1, 1998.

[20] F. Borgonovo, A. Capone, M. Cesana, and L. Fratta, "ADHOC MAC: a new, flexible and reliable MAC Architecture for ad hoc Networks," in IEEE WCNC'03, 2003.

[21] T. You, C.-H. Yeh, and H. Hassanein, "A new class of collisionprevention MAC protocols for ad hoc wireless networks," in IEEE ICC'03, 2003.

[22] H. Wu, A. Utgikar, and N.-F. Tzeng, "SYN-MAC: A Distributed Medium Access Control Protocol for Synchronized Wireless Networks," Springer J. Mobile Netw. Appl., vol. 10, 2005.

[23] Trimble Navigation Ltd., "Resolution T," 2004. [Online]. Available: http://www.trimble.com/resolutiont.shtml

[24] NavSync Ltd., "CW25-TIM Holdover Test," 2005. [Online]. Available: http://www.navsync.com/docs/AN03_GPS_Timing.pdf

[25] 802.11a-1999 (R2003), High-speed Physical Layer in the $5 \mathrm{GHz}$ Band, IEEE Std., 1999, Reaffirmed June 2003.

[26] A. V. Oppenheim, R. W. Schafer, and J. R. Buck, Discrete-time signal processing. Prentice Hall, 1999.

[27] C. Foh, , and J. Tantra, "Comments on IEEE 802.11 saturation throughput analysis with freezing of backoff counters," IEEE Commun. Lett., vol. 9, 2005.

[28] Atheros Communications, "AR5002X," 2003. [Online]. Available: http://www.atheros.com/pt/bulletins/AR5002X.pdf 